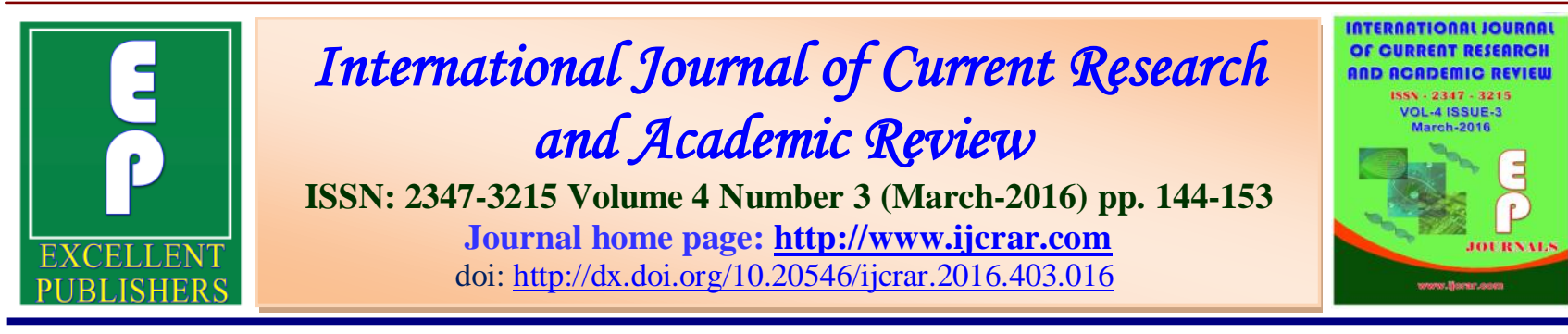

\title{
Green Nanomaterials: A Sustainable Development for Greener World
}

\author{
Ramesh Baboo*
}

Department of Chemistry, Govt. Girls P.G. College, Fatehpur, India

*Corresponding author

\begin{tabular}{l|l|}
\hline \multirow{2}{*}{$\begin{array}{l}\text { KEYWORDS } \\
\text { Nanotechnology, }\end{array}$} & A B S T R A C T \\
\cline { 2 - 3 } $\begin{array}{l}\text { Surface chemistry, } \\
\text { Nanomaterial. }\end{array}$ & $\begin{array}{l}\text { Besides numbers of commercial manufacturing attempts, nanoscience is } \\
\text { regarded as the key technology of the } 21^{\text {st }} \text { century. It provides novel products } \\
\text { and facilitates applications of innovative techniques in medicine, pharmacy, } \\
\text { computer technology, and sensing. At the same time, it is recognised that } \\
\text { their application may raise new challenges in the safety, regulatory or ethical } \\
\text { domains. Such challenges provide a new word, called Green nanoscience or } \\
\text { nanotechnology. It means applications of nanotechnologies must be } \\
\text { environmentally benign and sustainable. Use of nanotechnology in medicine } \\
\text { are especially promising, and areas such as disease diagnosis, drug delivery } \\
\text { targeted at specific sites in the body and molecular imaging are being } \\
\text { intensively investigated and some products are undergoing clinical trials. But } \\
\text { the area of health and medicines is more sensitive than others so the use of } \\
\text { sustainable and green nanotechnology reduces the risk of negative impacts on } \\
\text { environment, plant and animals. Many nanotechnologies pose no new risks to } \\
\text { health and almost all the concerns relate to the potential impacts of } \\
\text { deliberately manufactured nanoparticles and nanotubes that are free rather } \\
\text { than fixed to or within a material. Several studies have shown that altering the } \\
\text { surface chemistry of nanoparticles has been effective in preventing toxicity of } \\
\text { the core nanomaterials. }\end{array}$ \\
\hline
\end{tabular}

\section{Introduction}

Nanomaterials, with its peculiar dimension at the range of $1-100 \mathrm{~nm}$, are at the current edge of nanoscience and nanotechnology. Nanomaterials, specifically metal nanoparticles, have received particular attention in diverse field of applied science ranging from physical to biological sciences in past few years. The materials on the nanosize scale are different in chemical and physical properties than macrosized materials of identical or similar chemical composition. These materials can induce different biological responses. The type of biological response could also be due, in part, to the route of exposure and site of target tissues. Because these engineered nanomaterials are currently incorporated into a variety of industries (food packaging, 
forestry and paper, cosmetics, plastics and paints, and electronics) as composites, coatings, or suspensions, there is an overwhelming need to evaluate each nanomaterial. The synthesis and application of a range of functional nanomaterials promises to provide us with new tools in the fight against disease, climate change and the generation and storage of renewable energy. Scientists and engineers are therefore required to consider the consequences and implications of this type of nano tasking, and direct a delicate balance between technological advancement and social awareness.

The word green nanotechnology is just opposite to nanotoxicology. It means applications of nanotechnologies must be environmentally benign and sustainable. Thus the nanotoxicology or nanotoxicity is defined as, the potential of nanosized objects to cause harm to an organism, is a complex discipline, requiring the close collaboration between life and material sciences. In this context, it has become crucial to identify key parameters in the physico-chemical properties of nanomaterials that can affect toxicity, to make it possible for toxicology experiments to find the unique ways in which nanomaterials behave in standard toxicological media. Furthermore, in order to understand their impact on the environment and human health, the behaviour of nanomaterials has to be understood generically as well as in the context of toxicity experiments (Elsaesser and Howard, 2012) and based on that, further predictive modelling has to be developed for a comprehensive regulation to be established and for the safe development of nanotechnology (Burello and Worth, 2011). A further consideration for the successful implementation of nanotechnology is that of sustainability. A technology may be considered sustainable if it uses small amounts of limited resources, consumes minimal energy, causes minimum pollution and generates materials that can be recycled or reused. Nanotechnology can be developed successfully only if safe, green and cost-effective manufacturing lines are in place that allows one to obtain high quality, safe nanoproducts. If novel nanomaterials are not designed following these minimum requirements, they cannot be considered sustainable, and as a result their successful trade on large scale may be compromised.

The development of greener synthesis methods has helped in this way (Bayer et al., 2000). From pyrophoric precursors (Murray et al., 1993) to stable salts (Peng and Peng, 2000) and single source precursors (Malik et al., 2001), the use of less toxic capping and the reduction of waste generated (Liu et al., 2008) during nano dot synthesis was a breakthrough in the 1990s. Without overcoming these challenges, it would have been difficult to promote any development of nanocrystals. Hence, once the properties were optimised and the potential applications of nano dots established, most important concerns in the fabrication processes were altered. The use of nano dots was considered for green energy sources but how could this be promoted when the synthesis of the particles was not green or user-friendly? Not only will the particles have to be made using green synthesis (Raveendran et al., 2003) but the end product also needs to be safer. Another important step which needs understanding is the behaviour of nanoparticles in environmental and biological media (Lynch et al., 2009). The initial nanoparticles (primary nanoparticles) can be drastically changed in the biological system (secondary nanoparticles) which in the context of biomedical application can affect the properties that were initially desired. One of the first challenges in using nanoparticles for 
biological application was their hydrophobicity. Water-soluble nanoparticle synthesis was therefore investigated in order to obtain particles that exhibited the desired property in the biological medium considered. Similarly, the nanoparticles that are received by toxicologists (primary nanoparticles) are not the particles that are being tested for toxicity. The cells will be exposed to an altered version (secondary nanoparticles) that is the result of the interaction between the exposure medium and the primary nanoparticles.

Therefore, in parallel to studies aimed at optimizing biological and industrial performance, the toxicology of all types of nanomaterials is also under scrutiny (Dumortier et al., 2006; Magrez et al., 2006; Pulskamp et al., 2007), along with the consideration of possible environmental impacts (Donaldson et al., 2004; Lam et al., 2006; Barnard, 2006; Hyung et al., 2007) and issues surrounding workplace safety (Donaldson et al., 2006; Bartis and Landree, 2006). So, if one step back and take a broad view of the field, it can be seen that both the technological and the toxicological fields must be focused on the same goal and that is ensuring sustainability for the future of nanotechnology. There is increasing demand that new technologies be environmentally friendly, so that they do not damage the environment when discarded, when in use, or when in production (i.e. low carbon emissions). Ideally the new nanotechproducts to be recyclable and/or biodegradable, and of course, it is a need that nanotechnology must be safe. This means that the next generation of "indemand" smart products must be simultaneously efficient, safe and environmentally friendly and, above all else, they must be reliable and perform their function in a predictable way. This is a demanding resume, and in reality not all nanomaterials will meet these requirements.
In such cases it must be decided either to moderate, or acclimatize to preserve our quality of life.

While challenging, these issues are not entirely unfamiliar. In the past many nanoparticles were simply referred to as ultra-fine particles, which were known to either occur naturally (Guo and Barnard, 2012), or to be introduced unintentionally through human activities or as industrial products (Oberdo et al., 2005). The natural sources include gas-to-particle conversions, forest fires, volcanoes, viruses, biogenic sources, and the incidental sources include internal combustion engines, power plants, incinerators, jet engines, fumes (metal polymer, etc.), heated surfaces (such as in frying, boiling or grilling) and electric motors. In general, much of the study of hazards associated with nano-sized particles is based on established research on these airborne ultra-fine particles, and so are the assumptions. The main difference between this field and the emerging field of nanotoxicology is the issue of the nanomaterials being engineered. The nanomaterials engineered for the tasks mentioned above typically have no natural analogue, and are often artificially modified to induce a specific functionality. This introduces a degree of unpredictability, since a big range of largely untested materials are presented that are unique on an atomic scale. Unlike the nanomaterials from the natural and unintentional sources, intentionally produced nanoparticles (made in laboratories) have not been around long enough for public to collect sufficient data to estimate their long term potential for causing adverse effects.

Our only defense against the technological and environmental insecurity introduced by this cyclic causality is to increase our knowledge of the various types of 
instabilities native to nanomaterials, along with their respective causes. This includes structural instability, but also more subtle instabilities that may be more difficult to detect, such as conformational instabilities of impurities and functional defects or ligands adsorbed at the surfaces. The situation where impurities thought to be stable within the core of a nanoparticle were to diffuse to the surface, thereby changing the surface reactivity or causing stabilizing surfactants to desorb. As nanomaterials have a high surface area, maintaining stable surfaces both in devices and in storage media is of critical importance.

There is a clear need for short-term testing of the potential hazards of nanomaterials in order to gain information necessary to evaluate their risk. However, the large number of nanoparticles and the variety of their characteristics including sizes and coatings suggest that the only rational approach which avoids testing every single nanoparticle is to find a relationship between the physico-chemical characteristics of nanoparticles and their toxicity. Current risk-assessment paradigms, particularly in regulatory submissions for drugs and chemicals, generally depend on standardized methodologies.

\section{Initiatives towards Green Nanotechnology}

There is an unusual opportunity to use science, engineering and policy knowledge to design novel products that are benign as possible to human and environment health. Recognition of this opportunity has led to the development of the "green nanotechnology" concept (McKenzie and Hutchison, 2004; Dahl et al., 2007). Green nanotechnology has drawn on the field of green chemistry, and the framework of the 12 Principles of Green Chemistry (Anastas and Warner, 1998) features significantly in work to design new nanotechnologies for joint economic, social, and health/environmental benefit (Hutchison and Nano, 2008). These efforts have been aided by awareness throughout the nanotech community that they need to address the potential negative impacts of nano from the outset. In 2010, the first pilot summit was held in conjunction with the "Safer Nanomaterials and Nanomanufacturing's (SNNI)" Fifth Annual Conference in Portland, Oregon. The group of experts participating in the conference sessions has put the attention on four aspects in order to develop greener nanoscience or nanotechnology. First one is to find out the most important technical challenges. Second one is to understand the challenges of nanotoxicology and the associated informatics. Another one is about new policies, that are necessary to advance greener approaches to nanotechnology and last one is the most pressing industrial deployment challenges.

\section{Obstacles in the Development of Green Nanotechnology}

There are no clear designed guidelines for researchers in initial discovery phases of green nanoscience or nanotechnology. Many green nanomaterials require new commercial production techniques, which increases the need for basic research, engineering research, and coordination of the two between the industrial and research communities.

Another issue is the lack of a "deep bench" of scientists and engineers with experience developing green nanotechnology. It is most important aspect of green nanotechnology that the toxicity or toxicology and analysis protocols need to be developed and constantly updated to reflect advances in the 
Int.J.Curr.Res.Aca.Rev.2016; 4(3): 144-153

science. It is seen that the regulatory uncertainty persists, and green nanotechnologies often face higher regulatory barriers than existing or conventional chemicals. It is also observed that the end-market demand is unclear, especially since there are only a limited number of commercial grade products that can be compared to conventional materials in terms of performance.

\section{Conventional Physical and Chemical Synthesis of Nanomaterials}

Synthesis of Manufactured Nano Particles (MNPs) is carried out by several physical and chemical methods that include laser ablation, ion sputtering, (Raffi et al., 2007) solvothermal synthesis, (Rosemary and Pradeep, 2003) chemical reduction, and solgel method. Basically, there are two approaches for nanoparticle synthesis, the top-down and bottom-up. Top-down approaches seek to create nanoscale objects by using larger, externally controlled microscopic devices to direct their assembly, while bottom-up approaches adopt molecular components that are built up into more complex assemblies.

\section{Green Synthesis of Nanomaterials}

In terms of rendering nanomanufacturing processes less energy and resource intensive, the principles of Green Chemistry applied to nanotechnology involve, if at all possible, (a) the use of cost-effective, nontoxic precursors; (b) minimization of carcinogenic reagents and solvents (if possible, through utilization of aqueous solvents in order to bypass potentially toxic, acidic, or basic analogues); (c) reduction of experiments carried out with either pyrophoric compounds or unstable precursors to avoid risk; (d) use of relatively few numbers of reagents, i.e. atom economy, coupled with a conscious effort to circumvent the generation of greenhouse gases; (e) minimization of reaction steps leading to a reduction in waste, reagent use, and power consumption; (f) development of reactions to generate high-purity materials with little if any byproducts through high-yield processes; (g) ambient temperature and pressure synthesis, if at all possible, so as to preclude the need for either vacuum or high temperature processes; and (h) efficiency of scale-up. Parallel efforts in developing adequate characterization facilities as well as in promoting computer-aided nanomaterials modeling and process design tools have been of utmost importance in furthering all of these green objectives with the aim of creating environmentally benign building blocks and reducing the environmental footprint of manufacturing protocols (Diallo and Brinker, 2011). Optimally, green nanomanufacturing should use less material, less water, and less energy, and produce less waste as compared with conventional processes. Green Nanotechnology is an application that pays attention to implications. It is based on lowering the risk of using nanomaterials, limiting the risk of producing nanomaterials, and using nanomaterials to lower the risk of producing unwanted chemical intermediates and end-products. Green Nanotechnology maintains a commitment to rigor with applications related to sustainability as well as awareness about and prevention of possible harmful consequences from its successful implementation.

More in line with our spotlight on novel 'green' synthesis techniques, characteristic examples include recent successes in the development of template-assisted methods as well as the demonstration of proven protocols such as ultrasound and microwave irradiation specifically tailored for the production of novel, high-quality, 
multifunctional materials. In addition to technique development, there has been a growing emphasis on creating novel approaches towards greener synthesis to either (i) enhance the environmental friendliness of green synthetic techniques, ideally or (ii) serve as an alternative, when green synthetic techniques have not been practical. In this light, a number of fundamentally green approaches have included strategies such as substituting common reagents and solvents with more benign replacements such as biological reagents, ionic liquids, supercritical fluids, and even solvent-free methods.

Interestingly, citrate ions have been used as reducing agents, stabilizers, and complexing agents in the presence of surfactants to form Ag nanoplates, measuring from 100 to 350 nm under ambient conditions (Jiang et al., 2010). Highly stable, starch-stabilized aqueous dispersions of 1-8 $\mathrm{nm}$ nanoparticles of $\mathrm{Ag}, \mathrm{Au}$, and $\mathrm{Au}-\mathrm{Ag}$ alloys of varying compositions have also been synthesized in water using glucose as the reducing agent and starch as the protecting agent by microwave heating precursors for as little as $60 \mathrm{~s}$ in some cases (Raveendran et al., 2006). Macroscale quantities of bimetallic $\mathrm{Au}-\mathrm{Ag}$ alloy nanoclusters have been successfully achieved through simple mortar grinding of metal precursors in the presence of chitosan biopolymer, which was used as both a stabilizing and reducing agent (Murugadoss et al., 2012). Finally, bio-assisted syntheses of Ti nanospheres (40-60 nm)' (Prasad et al., 2007) anisotropic Au nanotriangles (25 to 35 $\mathrm{nm}$ ), (Verma et al., 2011) and $\mathrm{Pt}$ nanocrystals (including $4 \mathrm{~nm}$ polyhedra and 7-8 $\mathrm{nm}$ cubes) (Forbes et al., 2010) have been initiated using Lactobacillus, Aspergillus clavatus, and single peptide sequences, respectively. Simpler organisms, such as bacteria, algae, and fungi, have also developed highly specialized strategies for biominerals synthesis through hundreds of millions of years of evolution. The role of the templating molecule in biomineralization is to provide a synthetic microenvironment in which the inorganic phase morphology is tightly controlled by a range of low-range interactions. Among the microorganisms, prokaryotic bacteria have received the most attention in the area of metal nanoparticle biosynthesis. The formation of extracellular and intracellular metal nanoparticles by bacteria like Escherichia coli, Pseudomonas stutzeri, Pseudomonas aeruginosa, Plectonema boryanum, Salmonells typlus, Staphylococcus currens, Vibrio cholerae, etc., have been reported (Klaus et al., 1999). Extracellular secretion of the microorganisms offers the advantage of obtaining large quantities of materials in a relatively pure state, free from other cellular proteins associated with the organism with relatively simpler downstream processing. Mycelia free spent medium of the fungus, Cladosporium cladosporioides was used to synthesize silver nanoparticles extracellularly. It was hypothesized that proteins, polysaccharides and organic acids released by the fungus were able to differentiate different crystal shapes and were able to direct their growth into extended spherical crystals (Balaji et al., 2009). A number of plants are being currently investigated for their role in the synthesis of nanoparticles. Gold nanoparticles with a size range of $2-20 \mathrm{~nm}$ have been synthesized using the live alfalfa plants (Torresday et al., 2002). Nanoparticles of silver, nickel, cobalt, zinc and copper have also been synthesized inside the live plants of Brassica juncea (Indian mustard), Medicago sativa (Alfa lfa) and Heliantus annus (Sunflower). Certain plants are known to accumulate higher concentrations of metals compared to others and such plants are termed as hyperaccumulators. Of the plants 
investigated, B. juncea had better metal accumulating ability and later assimilating it as nanoparticles (Bali et al., 2006).

\section{Computational Design of Nanomaterials Prior to Synthesis}

More recently computer science has become involved in nanotechnology. Computational design of nanomaterial by using different softwares and computational methods can provide the information about the path and process of manufacturing and synthetic values without harming the environment. This provides the green path to understand the nanomaterial prior to synthesis at commercial as well domestic level. Such research is wide ranging and includes: software engineering, networking, internet security, image processing, virtual reality, human machine interface, artificial intelligence, and intelligent systems. Most work focuses on the development of research tools. For example, computer graphics and image processing have been used in nanomanipulators that provide researchers an interactive system interface to scanning-probe microscopes. Computer science offers more opportunities for nanotechnology. Soft Computing techniques such as swarm intelligence, genetic algorithms and cellular automata can enable systems with desirable emergent properties, for example growth, self-repair, and complex networks. Many researchers have successfully applied such techniques to realworld problems including complex control systems in manufacturing plants and air traffic control. With some modifications towards nanotechnology characteristics, these techniques can be applied to control a swarm of a trillion nanoassemblers or nanorobots. It is anticipated that soft computing methods such as these will overcome concerns about implications of nanotechnology, and prevent the notorious scenario of self-replicating nanorobots multiplying uncontrollably. Other current uses of computer science for nanotechnology include developing software systems for design and simulation. A research group at NASA has been developing a software system, called NanoDesign, for investigating fullerene nanotechnology and designing molecular machines. The software architecture of NanoDesign is designed to support and enable their group to develop complex simulated molecular machines. Computeraided nano-design (CAND) is an extension of com- puter-based engineering design traditionally at bulk scales to nanoscales. The general target of modeling and simulation in nano- materials design is to search stable and realizable structures and conformations with the minimal total system energy. At the molecular scale, parametric modeling mechanisms of particle aggregates are needed to support rapid construction and modification of geometries. Modeling behavior of complex physical and chemical systems, quantum effects in systems of nanoscopic dimensions, optical, magnetic, chemical and electrical properties of thin films, nanowires and quantum dots, electron transport in molecule-electrode interfaces, energy transfer in molecular assemblies, kinetics and energetics of elementary electron, computer simulations and experimental data are some current aspect of the computational science for the design of nanomatrials. Density functional theory provides a powerful tool for computations of the quantum state of atoms, molecules and solids, and of ab-initio molecular dynamics.

\section{Computational Testing of Toxicology as well as Biological Activity}

There is the potential for computational tools to refine, improve, and, in some cases, even replace existing tests. Computational 
tools are essential for increasing throughput, reducing the burden of animal testing, providing details of the toxicity mechanisms, and generating novel hypotheses for risk assessment (Veranthet al., 2007; Cengelli et al., 2006). For the purpose of nanoparticle assessment such approaches as the quantitative structureactivity relationship (QSAR) can be applied (Fond and Meyer, 2007; Gupta and Gupta, 2005; Hu et al., 2006). Computational tools are useful not only in making predictions, but also in refining existing risk-assessment paradigms. For example, QSAR approaches applied to assessing risk may facilitate the placement of chemicals with incomplete data sets in appropriate risk categories. Computational modeling includes physiologically-based pharmacokinetic (PBPK) models, as well as modeling doseresponses (Cengelli et al., 2006). If a QSAR model is then developed, ideally the doseresponse toxicity of untested nanoparticle can be predicted on the basis of its physicochemistry (Fond and Meyer, 2007; Gupta and Gupta, 2005; Gupta and Curtis, 2004). Actually, there is a strong need to extend the traditional QSAR paradigm to nanoparticles.

\section{Conclusion}

The current fast development of nanotechnology requires that nanotoxicology advances in parallel to allow regulation for the sake of public safety. Although there is not enough time to produce long and specialised experiments, well constructed and targeted experiments that can feed results into models could be the optimum approach. A very wide range of nanoparticle synthesis protocols have been developed in the past decades. Yet, when toxicity is the envisaged application of the nanoparticles, a selection has to be made assuring that the toxicity responses can be correctly interpreted. In fact, biosynthesis results in low energy use and environmental impact, with respect to conventional chemical synthesis methods. Further, the high specificity of biomolecules involved in the biosynthesis process may enable an efficient control of MNPs size and shape, whose tight control is critical to optimize MNP-based devices and applications. Current nanotoxicological research aims to identify the physico-chemical characteristics of NPs responsible for the observed health effects. These results could be incorporated in the design of new engineered NPs. The challenge is to produce new nanomaterials that are without adverse characteristics and still fulfil the industrial requirements. This approach would have the advantage of initiating a sustainable and safe nanotechnology. Many green nanomaterials require new commercial production techniques, which increases the need for basic research, engineering research, and coordination of the two between the industrial and research communities. Toxicology and analysis protocols need to be developed and constantly updated to reflect advances in the science.

\section{References}

Anastas, P.T., Warner, J.C. 1998. Green chemistry: theory and practice, Oxford University Press, Oxford England; New York.

Balaji, D.S., Basavaraja, S., Deshpande, R., Mahesh, D.B., Prabhakar, B.K., Venkataraman, A. 2009. Colloid Surf. B: Biointerfaces, 68: 88-92.

Bali, R., Razak, N., Lumb, A., Harris, A.T. 2006. IEEE Xplore.

Barnard, A.S. 2006. Nat. Mater., 5: 245.

Bartis, J., Landree, E. 2006. Nanomaterials in the Workplace: Policy and Planning Workshop on Occupational Safety and Health, RAND, Santa Monica, CA. 
Bayer, A., Boyle, D.S., Heinrich, M.R., O'Brien, P., Otway, D.J., Robbe, O. 2000. Green Chem., 2(2): 79-86.

Burello, E., Worth, A.P. 2011. Wiley Interdiscip. Rev., Nanomed. Nanobiotechnol., 3(3): 298-306.

Cengelli, F., Maysinger, D., TschudiMonnet, F., Montet, X., Corot, C., Petri-Fink, A., Hofmann, H., Juillerat-Jeanneret, L. 2006. J. Pharmacol. Exp. Ther., 318: 108116.

Dahl, J.A., Maddux, B.L.S., Hutchison, J.E. 2007. Chem. Rev., 107: 2228-2269.

Diallo, M., Brinker, C.J. 2011. Nanotechnology for Sustainability: Environment, Water, Food, And Climate. In Nanotechnology Research Directions for Societal Needs in 2020: Retrospective and Outlook; Roco, M., Mirkin, C. A., Hersam, M. C., Eds.; Springer Publishing: New York, 5: 157-187.

Donaldson, K., Aitken, R., Tran, L., Stone, V., Duffin, R., Forrest, G., Alexander, A. 2006. Toxicol. Sci., 92: 5 .

Donaldson, K., Stone, V., Tran, C.L., Kreyling, W., Borm, P.J.A. 2004. Occup. Environ. Med., 61: 727.

Dumortier, H., Lacotte, S., Pastorin, G., Marega, R., Wu, W., Bonifazi, D., Briand, J.P., Prato, M., Muller, S., Bianco, A. 2006. Nano Lett., 6: 1522.

Elsaesser, A., Howard, C.V. 2012. Adv. Drug Delivery Rev., 64(2): 129-137.

Fond, A.M., Meyer, G.J. 2007. (Nanomaterials - Toxicity, Health and Environmental Issues), Weinheim, Wiley-VCH, Vol. 5.

Forbes, L.M., Goodwin, A.P., Cha, J.N. 2010. Chem. Mater., 22: 6524-6528.

Gupta, A.K., Gupta, M. 2005. Biomaterials, 26: 1565-1573.
Gupta, K., Curtis, A.S. 2004. J. Mater. Sci. Mater. Med., 15: 493-496.

Hu, F., Neoh, K.G., Cen, L., Kang, E.T. 2006. Biomacromolecules, 7: 809816.

Hutchison, J.E. 2008. ACS Nano., 2: 395402.

Hyung, H., Fortner, J.D., Hughes, J.B., Kim, J.H. 2007. Environ. Sci. Technol., 41: 179.

Jiang, X.C., Chen, C.Y., Chen, W.M., Yu, A.B. 2010. Langmuir, 26(6): 44004408.

Klaus, T., Joerger, R., Olsson, E., Granqvist, C.G. 1999. Proc. Natl. Acad. Sci. U.S.A., 23(9624): 1361113614.

Lam, C.W., James, J.T., McCluskey, R., Arepalli, S., Hunter, R.L. 2006. Crit. Rev. Toxicol., 36: 189.

Liu, J.H., Fan, J.B., Gu, Z., Cui, J., Xu, X.B., Liang, Z.W., Luo, S.L., Zhu, M.Q. 2008. Langmuir, 24(10): 52415244.

Lynch, I., Salvati, A., Dawson, K.A. 2009. Nat. Nanotechnol., 4(9): 546-547.

Magrez, A., Kasas, S., Salicio, V., Pasquier, N., Seo, J.W., Celio, M., Catsicas, S., Schwaller, B., Forro, L. 2006. Nano Lett., 6: 1121.

Malik, M.A., Revaprasadu, N., O'Brien, P. 2001. Chem. Mater., 13(3): 913-920.

McKenzie, L.C., Hutchison, J.E. 2004. Chemistry Today, 25.

Murray, C.B., Norris, D.J., Bawendi, M.G. 1993. J. Am. Chem. Soc., 115(19): 8706-8715.

Murugadoss, A., Kai, N., Sakurai, H. 2012. Nanoscale, 4: 1280-1282.

Nature's Nanostructures. 2012. ed. H. B. Guo and A. S. Barnard, Pan Stanford Publishing, Singapore.

Oberdo rster, G., Oberdo rster, E., Oberdorster, J. 2005. Environ. Health Perspect., 113: 823. 
Peng, Z.A., Peng, X. 2000. J. Am. Chem. Soc., 123(1): 183-184.

Prasad, K., Jha, A.K., Kulkarni, A. 2007. Nanoscale Res. Lett., 2: 248-250.

Pulskamp, K., Diabat, S., Krug, H.F. 2007. Toxicol. Lett., 168: 58.

Raffi, M., Rumaiz, A.K., Hasan, M.M., Shah, S.I. 2007. J. Mater. Res., 22: 3378-3384.

Raveendran, P., Fu, J., Wallen, S.L. 2003. J. Am. Chem. Soc., 125(46): 1394013941.

Raveendran, P., Fu, J., Wallen, S.L. 2006. Green Chem., 8(1): 34-38.
Rosemary, M.J., Pradeep, T. 2003. J. Colloid Interface Sci., 268: 81-84.

Torresday, J.L.G., Parsons, J.G., Gomez, E., Peralta-Videa, J., Troian, H.E., Santiago, I.P., Yacaman, M.J. 2002. Nanolett., 2(4): 397-401.

Veranth, J.M., Kaser, E.G., Veranth, M.M., Koch, M., Yost, G.S. 2007. Fibre Toxicol., 4: 2.

Verma, V.C., Singh, S.K., Solanki, R., Prakash, S. 2011. Nanoscale Res. Lett., 6: 16/1-7.

\section{How to cite this article:}

Ramesh Baboo. 2016. Green Nanomaterials: A Sustainable Development for Greener World. Int.J.Curr.Res.Aca.Rev.4(3): 144-153. doi: http://dx.doi.org/10.20546/ijcrar.2016.403.016 